\title{
Evaluation of the ability of mice to detect VOCs, using a positive operant reinforcement procedure
}

\author{
Rieko Hojo \\ National Institute of Occupational Safety and Health, \\ Nagao 6-21-1, Tama-ku, Kawasaki city, Kanagawa 214-8585 Japan
}

(Received December 5, 2014; Accepted April 15, 2015)

\begin{abstract}
To examine the validity of a newly established "three-odor detection (TOD)" procedure using one of volatile organic compounds (VOCs), limonene, food-restricted male mice were used. Five animals each were assigned to either TOD or single-odor detection (SOD). TOD was composed of two trainings and one test (TEST) session. Mice were trained to discriminate an odor of coffee from no odor and odors of coffee and cheese from no odor in trainings 1 and 2, respectively. In TEST, mice were required to discriminate odors of coffee, cheese, and limonene from no odor. In SOD, mice were required to discriminate an odor of limonene from no odor. Each training or test was conducted once a day until animals achieved a learning criterion ( $75 \%$ correct response rate for 2 consecutive days), or until a maximum number of sessions ( 20 sessions) was completed. The number of sessions for reaching the learning criterion of animals in TEST ( $8.2 \pm 0.8)$ was smaller than that of animals in SOD (19.2 \pm 0.8$)$. Results indicated that mice in TOD detected low levels of VOCs more rapidly than animals in SOD. I concluded that TOD is a useful procedure for detecting low levels of VOCs.
\end{abstract}

Key words: Mouse, Three-odor detection (TOD), Operant behavior

\section{INTRODUCTION}

As many volatile organic compounds (VOCs) have typical smells, sometimes workers have reported that odors of VOCs induce adverse health problems or/and deprave symptoms (Berger-Sweeney et al., 1998) even if those concentrations were under the acceptable levels. As there are few studies focusing on the effects of the odor of VOCs per se on workers' health, causal relationships between health effects and the odors remain to be seen. To investigate the health effects of odors by animal experiments, it is essential to use test procedures that are able to estimate behavioral changes induced by odors. Many studies have demonstrated that mice readily learn tasks cued by odors, and they improve rapidly over successive trials (Singer, 2006) and show remarkable ability to detect odors (Wolkoff et al., 2006). For example, in the Go/No-go operant paradigm, mice have been found to reliably achieve high levels of detection accuracy across a variety of concentrations between 40-60 trials (Wolkoff et al., 2006) or within 100 trials (Zagreda et al., 1999). However, the focus in this research field was to explore the mechanism of the olfactory system in the animal, not to measure the odor threshold or odor detection ability in mice. In particular, there are a few problems with examining the odor detection ability of animals using VOCs: sometimes animals avoid sniffing VOCs because of the unfavorable smell; habituation or addictive effects of VOCs can also be problems for animals to examine the ability of odor detection or to measure the threshold of odors of VOCs. Aim of the present study was to develop an efficient odor detection procedure using animals without degrading the precision. A new procedure made it possible for mice to detect limonene, which is a VOC, within a small number of trials. Usually many trials were required to understand the relationship between odor discrimination and responding under odor discrimination experiments. Therefore it was difficult to use odor stimuli that have adverse effects for health, such as organic solvents. If the relationship is learned by a schedule controlled operant behavior (SCOB) using harmless odors in advance, learning to discriminate novel odors might be established with a smaller number of trials, even if they are noxious. In the present study, I established a new experimental procedure using odors, named three-odor detection (TOD). In the procedure, animals were trained

Correspondence: Rieko Hojo (E-mail: hojo@h.jniosh.go.jp) 
to discriminate between odor stimulus and 'no odor' by pressing levers. Then the relationship was generalized to a target (VOC) odor.

\section{MATERIALS AND METHODS}

C3H male mice (Japan Charles River, Tokyo, Japan) were housed individually under the following conditions: a 12-hr light/12-hr dark cycle (lights on at 7:00) at $21^{\circ} \mathrm{C}$ with free access to water. From 6 weeks of age, food was restricted to $2.5-4 \mathrm{~g}$ per day and maintained the mice at $80-85 \%$ of their free-feeding weights. Experiments were conducted in accordance with the Guidelines for the Care and Use of Animals of the National Institute for Environmental Studies (Tsukuba, Japan) and National Institute of Occupational Safety and Health (Kawasaki, Japan). All experiments were carried out using standard operant chambers for mice $(370 \mathrm{~W} \times 280 \mathrm{D} \times 280 \mathrm{mmH}$, Muromachi Kikai, Tokyo, Japan), equipped with two levers, two cue lights, a house light, a speaker for providing a tone, and a feeder. The chamber was individually located in a sound-attenuating box $(600 \mathrm{~W} \times 500 \mathrm{D} \times 500$ $\mathrm{mmH}$, Muromachi Kikai). Each stimulus was a tube containing the prepared odor, placed on a tray located under the floor of the chamber. Coffee and cheese, to which mice showed preference during a pilot study, were used as training odors in the TOD. Then limonene was selected as a test odor because mice strongly evaded it during the pilot study. As mice avoided the odor in the preliminary observation, it was thought that limonene was a good sample to examine the effect of our new odor detection procedure. Coffee, which was a commercial instant powder (Japan Nestle, Hyogo, Japan), was diluted with pure water to $1.0 \%$ concentration, which is available for drink. The cheese was a commercial cheddar paste (Kraft, Northfield, IL, USA) diluted with corn oil (Sigma-Aldrich, St Louis, MO, USA) to a concentration of $0.1 \mathrm{~g} / \mathrm{mL}$. Limonene (Sigma-Aldrich, Tokyo, Japan), diluted with pure water to a concentration of $1 \mu \mathrm{L} / \mathrm{mL}$, was used in TOD and SOD. These concentrations of coffee and cheese were selected because they could be spread in equal proportions on a cotton ball. Each odor stimulus, $300 \mu \mathrm{L}$ of volume, was absorbed into the cotton ball being put in the beneath of a $1.5 \mathrm{~mL}$ tube. The tube was covered with another cotton ball and then capped. Tubes were wrapped with white paper to eliminate visual cues. The tube was uncapped to release the odor immediately before putting the tube in a small dish, and then presented on a tray located under the grid floor of the chamber. Two weeks after the start of deprivation, 10 mice were engaged in the magazine training. At 10 weeks of age, 5 animals each were assigned to the TOD and the SOD. The TOD was comprised of three parts: trainings 1 (T1), 2 (T2), and a test (TEST). In T1, animals were required to press one of two levers, which corresponded to coffee odor or no odor. In T2, cheese was added to odor stimuli. In TEST, animals were required to press the odor lever when any of three odors, coffee, cheese or limonene was presented. SOD simply required animals to discriminate between limonene and no odor respectively. In $\mathrm{T} 1$ and $\mathrm{T} 2$, odor and no odor trials were shown 50 times each. TEST contained 25 odor and 25 no odor trials. The order of stimulus presentation in both TOD and SOD was randomized.

Each animal was engaged 1 session/day, 5 days/week during 20 sessions. At the beginning of a session, the house light was on and a tone was sounded for $0.5 \mathrm{sec}$ before each trial. A stimulus was manually presented approximately $0.5 \mathrm{sec}$ after the tone. Cue lights above the levers were on after the animal sniffed the stimulus for $2 \mathrm{sec}$, and levers were activated. When the animal selected the correct lever, a $14 \mathrm{mg}$ pellet (Bio-Serv, Flemington, NJ, USA) was delivered. On the other hand, if the animal selected the wrong lever or did not respond after more than $30 \mathrm{sec}$, the trial was terminated, house and cue lights, and levers turned off. In $30 \mathrm{sec}$ intervals between trials, air of the chamber was flushed by an electric fan, and the dish and the tray were wiped with a wet paper towel. Correct response rate of each session was calculated. The session was conducted until the animal reached $75 \%$ correct in 2 consecutive sessions. The number of sessions needed to reach the criteria was defined as a learning load index (LLI). The average LLI of TOD was compared with that of SOD and was analyzed by the Student's t-test. The LLIs were verified by statistically significant increases in correct response rate at final sessions compared to those at the starting sessions by a two-tailed Student's t-test. All statistical analyses were performed with SPSS statistical package 19.0 software (IBM, Chicago, IL, USA). A $P$-value of $\leq 0.05$ was considered significant.

\section{RESULTS}

In SOD, the average LLI was $19.2 \pm 0.8$ (Table 1). The range of LLIs was 18-20, the upper limit of the range exceeded 20 because 2 mice out of 5 could not reach the criteria within 20 sessions. Even though some animals did not reach the criteria, the average correct response rate in session $20(68.3 \pm 11.6)$ was significantly higher than that of the first session (19.5 \pm 18.1$)$ (Fig. 1), it meant that animals achieved the task as a group. The average correct response rates of limonene $(54.3 \pm 12.3)$ and no odor $(61.0 \pm 14.3)$ were not different. In T1, all animals reached 
Evaluation of the ability of mice to detect VOCs

Table 1. Average number and range of sessions required to reach the $75 \%$ correct response rate.

\begin{tabular}{|c|c|c|c|c|c|c|}
\hline & & & $\begin{array}{l}\text { Number of sessions for reaching } \\
\text { the learning criterion (sessions) }\end{array}$ & $\begin{array}{l}\text { Learning load index } \\
\text { (sessions) }\end{array}$ & Cor & $\begin{array}{l}\text { nse rate of } \\
\text { lus }(\%)\end{array}$ \\
\hline \multirow{4}{*}{ Procedure } & \multicolumn{2}{|l|}{ SOD } & $19.2 \pm 0.8$ & $18-20$ & $\begin{array}{l}\text { Limonene } \\
\text { No odor }\end{array}$ & $\begin{array}{r}54.3 \pm 12.3 \\
61 \pm 14.3\end{array}$ \\
\hline & \multirow{3}{*}{ TOD } & $\mathrm{T} 1$ & $6.8 \pm 0.2^{*}$ & $6-7^{*}$ & $\begin{array}{l}\text { Coffee } \\
\text { No odor }\end{array}$ & $\begin{array}{l}75.4 \pm 3.5 \\
65.3 \pm 3.5\end{array}$ \\
\hline & & $\mathrm{T} 2$ & $18.2 \pm 1.3$ & $16-19$ & $\begin{array}{l}\text { Coffee } \\
\text { Cheese } \\
\text { No odor }\end{array}$ & $\begin{aligned} 75 & \pm 2.7^{\mathrm{a}, \mathrm{b}} \\
59.9 & \pm 3 \\
65.6 & \pm 3.3\end{aligned}$ \\
\hline & & $\mathrm{T} 3$ & $8.2 \pm 0.8^{*}$ & $7-9^{*}$ & $\begin{array}{c}\text { Coffee } \\
\text { Cheese } \\
\text { Limonene } \\
\text { No odor }\end{array}$ & $\begin{aligned} 82 & \pm 7.8^{\mathrm{a}, \mathrm{c}} \\
85 & \pm 7.1^{\mathrm{a}, \mathrm{c}} \\
63.1 & \pm 8 \\
64.5 & \pm 2.5\end{aligned}$ \\
\hline
\end{tabular}

* means $<0.05$ (vs. SOD), ${ }^{\text {a }}$ means $<0.05$ (vs. no odor), ${ }^{\text {b }}$ means $<0.05$ (vs. cheese), ${ }^{\mathrm{c}}$ means $<0.05$ (vs. limonene) in the same procedure.

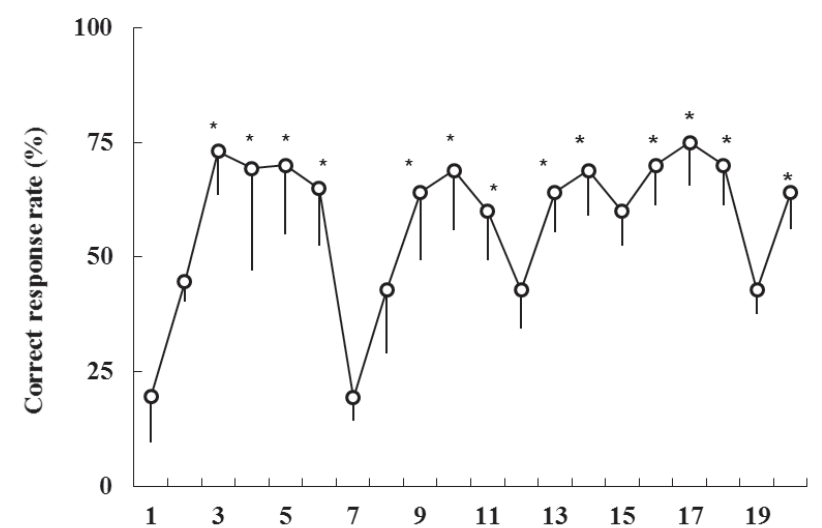

Fig. 1. Correct response rate (mean \pm S.E.M) of mice for limonene at SOD. $(*=p<0.05$, vs. correct response rate in the first session).

the correct response rate within the range of 6-7 sessions (Table 1). The average number of session required a $75 \%$ correct rate $(6.8 \pm 0.2)$, which was significantly lower than that of SOD. The average correct response rate of coffee $(75.4 \pm 3.5)$ in $\mathrm{T} 1$ was significantly higher than that of no odor (65.3 \pm 3.5 ) (Fig. 2A). In T2, animals took 16-19 sessions to reach a $75 \%$ correct rate. The average number of sessions $(18.2 \pm 1.3)$ was significantly higher than that of T1; however, it was not different from that of SOD (Table 1). The average correct response rate of coffee $(75.0 \pm 2.7)$ in $\mathrm{T} 2$ was significantly higher than those of cheese $(59.9 \pm 3.0)$ and no odor $(65.6 \pm 3.3)$ (Fig. 2B and C). These results implied that animals showed more difficulty in learning to press the same lever for coffee and cheese, even if these odors were presented differently. The correct response rates of coffee were higher in almost all trials than those of no odor at T2, even though both stimuli were experienced in the same amounts of trials in T1 and T2. In TEST, the number of sessions $(8.2 \pm 0.8)$ required to reach a $75 \%$ correct rate was significantly lower than those of SOD and T2, but not different from that of $\mathrm{T} 1$. The range of sessions to reach a $75 \%$ correct rate was 7-9 (Table 1). Fig. 2D and E show the correct response rate for odor and no odor stimulus during TEST, respectively. The average correct response rate for coffee $(82.0 \pm 7.8)$ and cheese $(85.0 \pm 7.1)$ were significantly higher than those of limonene $(63.1 \pm 8.0)$ and no odor $(64.5 \pm 2.5)$ in T2. Also, correct rates of coffee and cheese in sessions 2,4 , and 5 were higher than those of no odor $(69.2 \pm 4.22,52.5 \pm 3.02$, and $55.0 \pm$ 3.12 at sessions 2,4 , and 5). The average number of correct rates of coffee $(82.0 \pm 7.8)$ and cheese $(85.0 \pm 7.1)$ were greater than those of limonene $(63.1 \pm 8.0)$ and no odor $(64.5 \pm 2.5)$. The stimulus of no odor did not reach a $75 \%$ response rate until the last session.

\section{DISCUSSION}

I examined sensitivity to odor of mice using limonene as one of VOCs and evaluated effectiveness and validity of TOD. The main finding in this study is that limonene was readily detected by mice in TOD. In the Go/Nogo operant paradigm, mice have been found to reliably achieve high levels of detection accuracy across a variety of concentrations between 40-100 trials (Wolkoff et al., 2006; Zagreda et al., 1999). In the present study, the total number of trials of limonene detection that achieved the learning criterion in TOD was 68.3 (7-9 trials/session). The ability of odor detection in mice shown in the present study is comparable to the results of other studies that evaluated olfactory learning (Huttera et al., 2006). I concluded that TOD using mice might be one of useful 


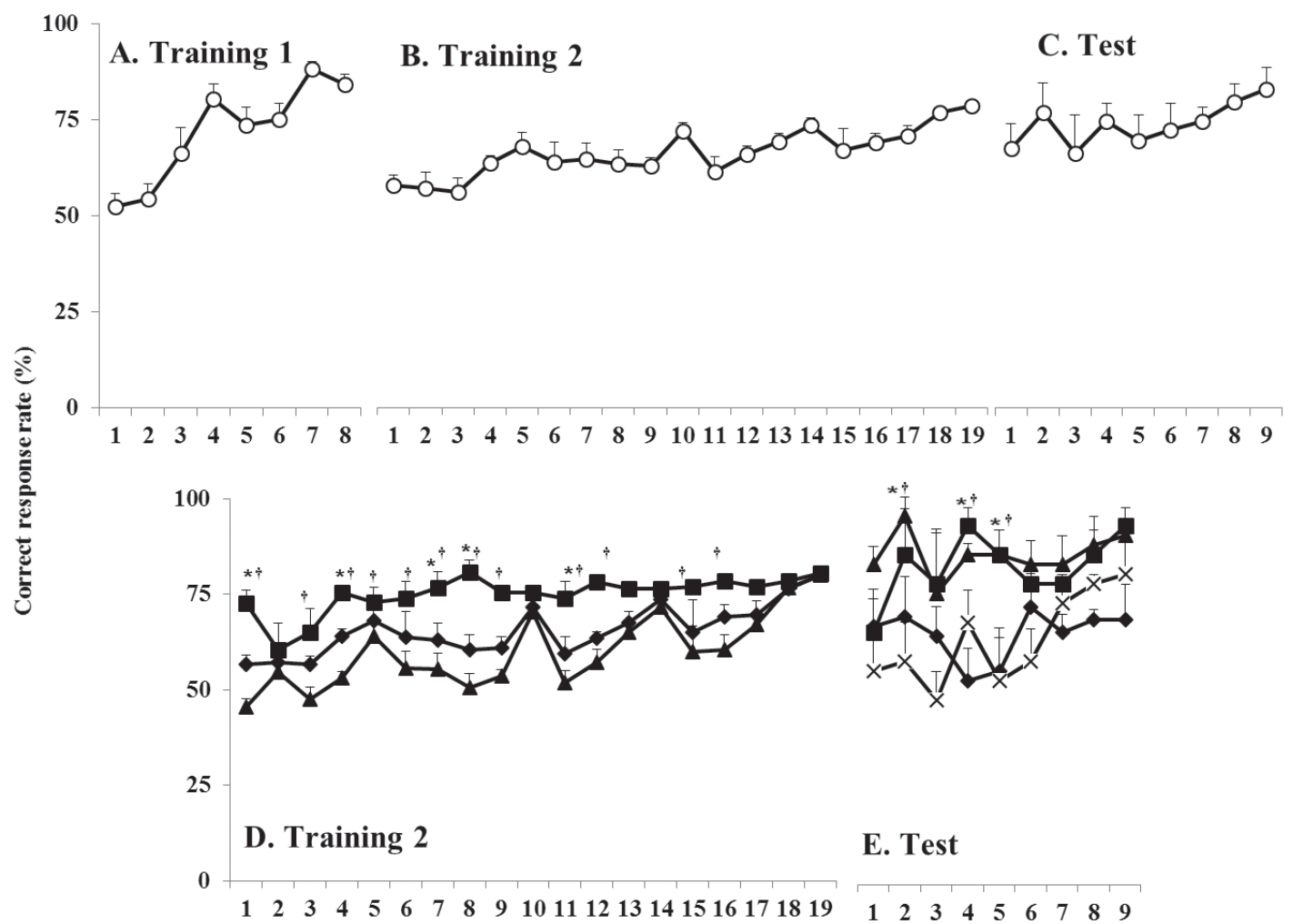

Fig. 2. Group means of correct response rates of mice at training 1 (A), 2 (B) and Test (C), and correct response rates of each odor and no-odor stimuli (D) in training 2 and Test (E). Correct response rates of group mean (O), coffee ( $(\mathbf{)})$, cheese $(\boldsymbol{\Delta})$, limonene $(\times)$, and no-odor $(\diamond)$ are shown. All data are presented as means \pm S.E.M. $\quad(*=p<0.05$, vs. correct response rate in the first session, $\uparrow=p<0.05$, vs. correct response rate in the first session in SOD).

methods for detecting odor of VOCs. The American Conference of Governmental Industrial Hygienists (ACGIH) indicated the threshold limit value for a variety of VOCs in the workplace. Though Threshold Limit Value (TLV) for limonene is not included in the ACGIH's guidelines, levels may easily reach several hundred parts per billion (ppb) during and immediately after product use (Walker and O'Connell, 1986; Wainman et al., 2000). Seifert et al. (1999) indicated $0.03 \mathrm{mg} / \mathrm{m}^{3}$ as guideline for terpenes in the guideline values for indoor air pollutant. The volume of limonene used in the TEST of the present study was $0.84 \mu \mathrm{g}(1 \mu \mathrm{L})$ in the $1.5 \mathrm{~m}^{3}$-sound attenuating box, and the concentration was $\left.0.56 \mu \mathrm{g} / \mathrm{m}^{3}\right)$. This concentration could be thought very low level as compared to the guideline value (Seifert et al., 1999). I concluded that TOD is useful to detect low levels of VOCs in indoor air for risk assessment. The number of sessions required to reach a correct response was set at $75 \%$ in the present study, which was lower than in other studies; for example, $90 \%$ (Wolkoff et al., 2006) and 80\% (Nakayama and Morimoto,
2009) were set in the Go/No go experiment paradigm used by other investigators. In all procedures of the present study, the correct response rates in the last session were higher than those in the first session. This means that I can suggest that animals learn odor detection in the process of these procedures, and a $75 \%$ correct rate was enough to reason that animals had learned the odor detection. The accuracies of odor detection in the present study were unstable, as indicated when they were decreased at sessions 5-7 in the experiment. Differences in accuracy can be due to procedural differences. For acquisition of associative learning, the salience of the stimulus cues is an important factor in determining how well the task is learned (Iversen et al., 1986). If stimulus cues are salient to evoke a response, conditional discrimination develops earlier and more accurately than when the cues are less salient (Iversen et al., 1986). In the present study, mice had to learn to press levers not only for odor stimuli but also for no odor. Responding to an undetectable stimulus could be difficult for mice from an associative learning 
Evaluation of the ability of mice to detect VOCs

standpoint. No odor stimulus might be difficult to evoke a response because no odor would not function as a discrimination cue. Therefore, I thought that the $75 \%$ correct rate set in the present study was not lower than the other studies.

It is assumed that the animals tried to distinguish the very low level of smells in the no odor stimulus because the rate of correct responses was lower than that of the other odor stimuli. In TOD, mice learned the relationship between stimuli (odor)-response (specific lever pressing)reward (food pellets for correct responding) during training. However, there were some problems in TOD procedure. Animals needed a lot of trials in T2 to reach the learning criterion. Animals might need time to categorize two stimuli (coffee and cheese) as odor stimuli, at the same time they might distinguish no odor as different stimulus from odor stimuli. In addition, at the beginning of TEST, correct response rates of limonene and no odor were not stable. It was thought that animals tried to categorize three odors as odor stimulus.

In the TEST session of TOD, the number of sessions to reach the criteria was significantly smaller than that in SOD. If mice associated each odor stimulus and response one by one when the conditioning was established, the results of SOD should be similar to the results of TOD. These results support that mice applied the response-behavior relationship acquired in the training to TEST as a category and did not use each odor as a simple element. Therefore, I concluded that TOD can be applied to testing other chemicals that have adverse effects, such as toxic, hedonic, or addictive effects.

In summary, mice were able to detect low levels of VOCs when trained by positive reinforcement in an operant procedure. TOD may be applicable to testing for other harmful chemicals because it minimizes exposure time.
Conflict of interest---- The authors declare that there is no conflict of interest.

\section{REFERENCES}

Berger-Sweeney, J., Libbey, M., Arters, J., Junagadhwalla, M. and Hohmann, C.F. (1998): Neonatal monoaminergic depletion in mice (Mus musculus) improves performance of a novel odor discrimination task. Behav. Neurosci., 112, 1318-1326.

Huttera, H.-P., Moshammera, H., Wallnerb, P., Dambergerc, B., Tapplercd, P. and Kundia, M. (2006): Health complaints and annoyances after moving into a new office building: A multidisciplinary approach including analysis of questionnaires, air and house dust samples. Int. J. Hyg. Environ. Health, 209, 65-68.

Iversen, I.H., Sidman, M. and Carrigan, P. (1986): Stimulus definition in conditional discriminations. J. Exp. Anal. Behav., 45, 297-304.

Nakayama, K. and Morimoto, K. (2009): Risk factor for lifestyle and way of living for symptoms of sick building syndrome: Epidemiological survey in Japan. Jpn. J. Hyg., 64, 689-698.

Seifert, B., Englert, N., Sagunski, H. and Witten, J. (1999): Regulationg indoor air. In Indoor air 99: Proceedings of the $8^{\text {th }}$ international conference on indoor air quality and climate. Guideline values for indoor air pollutants. Vol. 1, 499-504.

Singer, B.C., Destaillats, H., Hodgson, A.T. and Nazaroff, W.W. (2006): Cleaning products and air fresheners: emissions and resulting concentrations of glycol ethers and terpenoids Indoor Air, 16, 179-191.

Wainman, T., Zhang, J., Weschler, C.J. and Lioy, P.J. (2000): Ozone and limonene in indoor air: a source of submicron particle exposure. Environ. Health. Perspect., 108, 1139-1145.

Walker, J.C. and O'Connell, R.J. (1986): Computerized odor psychophysical testing in mice. Chem. Senses, 11, 439-453.

Wolkoff, P., Wilkins, C.K., Clausen, P.A. and Nielsen, G.D. (2006): Organic compounds in office environments - sensory irritation, odor, measurements and the role of reactive chemistry. Indoor Air, 16, 7-19.

Zagreda, L., Goodman, J., Druin, D.P., McDonald, D. and Diamond, A. (1999): Cognitive deficits in a genetic mouse model of the most common biochemical cause of human mental retardation. J. Neurosci., 19, 6175-6182. 